\title{
VI. Die „Reichswesthilfe“ im Dienste der Politik des Nationalsozialismus
}

\section{Die Ausschaltung der Länder durch „Gleichschaltung“}

Bis zum Frühjahr 1933 beruhte die Westhilfe auf einer Zusammenarbeit zwischen den Reichsministerien für Finanzen, Inneres und - zeitweise - für die besetzten Gebiete auf der einen und den Regierungen der Länder PreuBen, Bayern, Baden, Hessen, Oldenburg auf der anderen Seite. Die Entstehung und die Entwicklung des Westprogrammes boten im großen und ganzen das Bild einer sinnvollen Verbindung zwischen Unitarismus und Föderalismus. Die Schwächen des föderalistischen Staatsaufbaus, die sich im Bereich der Wirtschaftspolitik in einer Uneinigkeit der einzelnen Länder über die anzustrebenden Ziele und über die zu ergreifenden Maßnahmen offenbarten ${ }^{1}$, überdeckte das Reich bei der Westhilfe durch die zentrale Verteilung der finanziellen Mittel. Andererseits nutzte es die größere Sachkenntnis der Landesregierungen bei der Aufstellung und der Durchführung der einzelnen Arbeitsvorhaben. Diese ungeachtet gelegentlicher Auseinandersetzungen zwischen Reich und Ländern über die Höhe der Fondsmittel und das Verfahren der Verteilung doch recht fruchtbare Zusammenarbeit zerstörte kurz nach der nationalsozialistischen Machtergreifung die „Gleichschaltung“. Das am 31. März 1933 erlassene erste Gesetz zur Gleichschaltung der Länder mit dem Reich behielt zwar die Länderparlamente bei, forderte aber deren Neubesetzung nach Maßgabe der Verteilung der Wählerstimmen auf die einzelnen Parteien, wie sie sich bei den Wahlen zum Reichstag am 5. März ergeben hatte. Obendrein wurde die Gesamtzahl der Mandate um die Sitze verringert, welche die KPD errungen hatte. Gleichzeitig ermächtigte das Gesetz die Landesregierungen, ohne vorhergehende Beschlußfassung der Landtage selbständig verfassungsändernde Gesetze zu erlassen und die Landesverwaltung neu zu ordnen. ${ }^{2}$ Ein zweites Gesetz zur Gleichschaltung der Länder mit dem Reich, das am 7. April verkündet wurde, schuf in allen Ländern, Preußen ausgenommen, das Amt des Reichsstatthalters, dessen Träger vom Führer und Reichskanzler Adolf Hitler selbst vorgeschlagen und ernannt wurden. ${ }^{3}$ Der Reichsstatthalter erhielt den Auftrag, die jeweilige Landesregierung zu beaufsichtigen und die Einhaltung der Politik der

\footnotetext{
1 Siehe hierzu H. Klöter, Der Anteil der Länder an der Wirtschaftspolitik der Weimarer Republik 1919-1933, 1967, S. 231.

2 RGBl. I, 1933, S. $153 \mathrm{f}$.

3 Ebd., S. 173.
} 
Reichsregierung zu überwachen. Außerdem übertrug ihm der Führer das Recht der Ernennung und der Entlassung der Landesregierung, die Befugnis zur Auflösung des Landtags sowie das Recht zur Ausfertigung und zur Verkündigung der Landesgesetze. ${ }^{4}$ Ihren Abschluß fand die Gleichschaltung durch das „Gesetz über den Neuaufbau des Reichs" vom 30. Januar 1934, das die Länderministerien den Anweisungen des Reichsinnenministers unterstellte. ${ }^{5}$ Stolz verkündeten die Nationalsozialisten: „Auch der Länder„Patriotismus" ist nun nicht mehr in der Lage, Pläne und Maßnahmen der Reichsregierung zu durchkreuzen, wie es z. B. die sozialistische preußische Regierung jahrelang in den deutschen Ostfragen getan hat."6

In den am Westprogramm beteiligten Reichsländern bewirkte die Gleichschaltung die folgende Umgruppierung in der politischen Führung: In Preußen wurde am 11. April 1933 Hermann Göring zum Ministerpräsidenten ernannt, nachdem bis zur Machtübernahme Kurt von Schleicher und hernach Franz von Papen das Amt des Reichskommissars ausgeübt hatten. ${ }^{7}$ In Bayern ernannte Reichsinnenminister Frick am 10. April den Reichsleiter der NSDAP, Franz von Epp, zum Reichskommissar, der das seit dem 20. August 1931 nur noch geschäftsführend im Amt befindliche Kabinett Held ablöste. ${ }^{8}$ Anfang Mai stieg v. Epp zum Reichsstatthalter auf. ${ }^{9}$ In Baden trat die letzte demokratisch gewählte Regierung, eine Koalition aus Zentrum und DVP, am 10. März 1933 zurück, nachdem Frick zwei Tage zuvor den Gauleiter Robert Wagner zum Reichskommissar für das Polizeiwesen in Baden ernannt hatte. Am 5. Mai wurde Wagner Reichsstatthalter und übergab dem stellvertretenden Gauleiter Köhler die Ämter des Ministerpräsidenten, des Finanz- und des Wirtschaftsministers. ${ }^{10}$ In Hessen wurde schon am 6. März Heinrich Müller als Reichskommissar eingesetzt, den wiederum der Gauleiter von Hessen-Nassau, Jakob Sprenger, im Mai als Reichsstatthalter ablöste. ${ }^{11}$ In Oldenburg bescherte bereits die Landtagswahl vom 29. Mai 1932 der NSDAP die absolute Mehrheit, so daß der ,alte Kämpfer“ Carl Röver legal die Regierung übernahm. Nach der Gleichschaltung ernannte ihn Hitler im Mai zum Reichsstatthalter von Oldenburg und Bremen. ${ }^{12}$

4 Vgl. Hüttenberger, Die Gauleiter. Studie zum Wandel des Machtgefüges in der NSDAP, 1969, S. 76.

5 RGB1. I, 1934, S. 75. In diesem Gesetz wurden ferner die Volksvertretungen der Länder aufgehoben und die Hoheitsrechte der Länder auf das Reich übertragen.

6 P. Hövel, Grundfragen deutscher Wirtschaftspolitik, 1935, S. 27.

7 Hubatsch, S. 88.

8 Zur nationalsozialistischen Machtübernahme in Bayern siehe Wiesemann, S. $254 \mathrm{f}$.

9 E. Deuerlein, Bayern, in: Territorien-Ploetz 2, S. $401 \mathrm{f}$.

10 Haselier, S. 467f.; ferner H. Rehberger, Die Gleichschaltung des Landes Baden 1932/ 33, 1966.

11 Demandt, S. 499.

12 G. Schnath u. a., Niedersachsen, in: Territorien-Ploetz 2, S. 583. 
Hatten noch im Februar die Vertreter der Länder versucht, der Reichsregierung, die nunmehr durch das erste Kabinett Hitlers verkörpert wurde, ihre Vorstellungen zur Gestaltung der künftigen Westhilfe nahezubringen, ${ }^{13}$ so sahen sie sich bereits im April von der Mitwirkung an der Planung des Westprogramms abgeschnitten. Daß diese Entwicklung nicht von vornherein im Sinne der Gefolgsleute Hitlers lag, die mittlerweile die Länderministerien erobert hatten, bezeugt deren Bemühen um die Beibehaltung eines gewissen Mitspracherechts. So suchte der nationalsozialistische Ministerpräsident Hessens, Dr. Werner, ${ }^{14}$ am 21. April bei Frick um eine Beteiligung seines Landes an der zu erwartenden RWH 1933 nach und entwarf gleichzeitig seine Vorstellungen über die Höhe des Anteils, den er für sein Land angemessen hielt. Werner verwies, darin seinen demokratischen Vorgängern folgend, auf die Besatzungsschäden, die Hessen in außergewöhnlichem Maße betroffen hätten und die seine Steuerkraft immer noch beeinträchtigten. ${ }^{15}$ Daraufhin vertröstete ihn Frick mit den kommenden ,großen“ Arbeitsbeschaffungsprogrammen des Reiches. Hartnäckig hielt Werner jedoch an seinem Hilfegesuch fest. Zwischen den Mitteln der Arbeitsbeschaffung und den Geldern der Westhilfe bestünden große Unterschiede, belehrte er Frick am 9. Juni, denn diese würden in der Regel als verlorene Zuschüsse des Reiches gewährt, während jene nur Darlehen zu günstigen Zinsbedingungen darstellten. Hessen könne sich indessen in seiner großen wirtschaftlichen Not keine neue Schuldenaufnahme mehr erlauben. Erst kürzlich habe die „Öffa“16 nämlich der Stadt Mainz Kredite aus dem ,Sofortprogramm“ verweigert, mit dem Hinweis auf noch zu tilgende Darlehen, welche die Gemeinde bereits früher aufgenommen habe. Gerade im Raum Mainz habe jedoch die Westhilfe der vergangenen Jahre eine spürbare Belebung der Wirtschaft bewirkt. ${ }^{17}$ Das Lob, das Werner hier dem Westprogramm der Republik zollte, beeindruckte den Reichsminister nicht. Kühl erwiderte Frick, mit Rücksicht auf die kommende Saarabstimmung werde sich die RWH 1933 ausschließlich auf den Saargrenzgürtel beschränken. Wenn er auch durchaus verstehe, daß man in Hessen den Fortfall der Westhilfe schmerzlich empfinde, so

13 GLA 233, Nr. 26040, 16.2., 21.2., 28.2.33; BHStA MA 106079, 28.2.33.

$14 \mathrm{Zu}$ den Auseinandersetzungen über die Verteilung der Macht in Hessen zwischen Ministerpräsident und Reichsstatthalter vgl. P. Diehl-Thiele, Partei und Staat im Dritten Reich. Untersuchungen zum Verhältnis von NSDAP und allgemeiner innerer Staatsverwaltung 1933-1945, 1969, S. $50 \mathrm{f}$.

15 BA R 43 II/127a, Bl. 3-6.

16 Diese staatliche Bank übernahm ab 1930 die Aufgaben des Reiches auf dem Gebiete der wertschaffenden Arbeitslosenfürsorge. Sie wurde 1932 zum hauptsächlichen Träger der großen Arbeitsbeschaffungsprogramme der Reichsregierung. K. Seifert, Art. „Deutsche Gesellschaft für öffentliche Arbeiten AG (Öffa)“, in: Enzyklopäd. Lexikon, S. 385.

17 BA R 43 II/127a, Bl. 9-11. 
erwarte er dennoch, , daß das Land Hessen im Hinblick auf das zu erstrebende große Ziel und unter Zurücksetzung eigener berechtigter Wünsche die Gründe meiner Entschließung würdigen wird." 18

Auch Josef Bürckel, der Gauleiter der Rheinpfalz, setzte sich für eine angemessene Beteiligung des von ihm beherrschten Landesteils bei der RWH 1933 ein, wobei er geschickt seine persönlichen politischen Vorstellungen und wirtschaftlichen Zielsetzungen miteinander verknüpfte. Stets hatte Bürckel den sozialistischen Ausgangspunkt der „Bewegung“ betont. Gemeinsam mit dem Hamburger Gauleiter Kaufmann gehörte er zu den entschlossensten Verfechtern der ,populistischen“ Richtung im Nationalsozialismus. In Fragen, welche die Lebenshaltung der Bevölkerung betrafen, scheute er auch die offene Auseinandersetzung mit Reichsarbeitsminister Franz Seldte und dem einflußreichen, ab August 1934 als Reichswirtschaftsminister amtierenden Hjalmar Schacht nicht. ${ }^{19}$ Außerdem aber zeigte sich Bürckel schon früh als Exponent einer „Los-von-München“-Bewegung, welcher die pfälzischen Sonderinteressen gegen München genau so hartnäckig verteidigte, wie dies die bayerischen Regierungsstellen gegenüber dem Reich taten. ${ }^{20}$ Vom Reichsinnenminister erwartete er deshalb die Durchführung von Maßnahmen der Arbeitsbeschaffung und die bevorzugte Vergabe öffentlicher Aufträge an die pfälzische Wirtschaft. Schließlich verlangte er, hierin ebenfalls dem Vorbild demokratischer Politiker nacheifernd, Frick möge die Westhilfe nach dem Beispiel der Osthilfe auf eine gesetzliche Grundlage stellen. Frick indessen ließ es bei einer Informationsreise bewenden, die einer seiner Mitarbeiter in die Pfalz unternahm. ${ }^{21}$ Am 18. Juli 1934 löste Bürckel den bisherigen Reichsbevollmächtigten für das Saargebiet, v.Papen, ab. ${ }^{22}$ Mit der Úbernahme dieses Amtes wurden seine Westinteressen mit denen der Regierung in Berlin automatisch, gleichgeschaltet". Bald darauf fanden sie ihren Niederschlag sogar in einer offiziösen ,Westmark"-Ideologie. ${ }^{23}$

Doch nicht allein auf den Umfang einer künftigen Reichswesthilfe hatten die von Nationalsozialisten geführten Landesregierungen keinen Einfluß mehr. Auch die Verteilung der noch vorhandenen Gelder aus den Westfonds der Jahre 1930 bis 1932 wurde nun von Berlin aus gesteuert. Wo immer es möglich schien, lenkte der Reichsinnenminister diese Mittel in Verwendungen, die eine Ergänzung zur „Winterarbeitsbeschaffung“ der Reichsregie-

18 Ebd., Bl. 17.

19 T. W. Mason, Arbeiterklasse und Volksgemeinschaft. Dokumente und Materialien zur deutschen Arbeiterpolitik 1936-1939, 1975, S. 96f.; Hüttenberger, Gauleiter, S. 140.

20 O. Domröse, Der NS-Staat in Bayern von der Machtergreifung bis zum Röhm-Putsch, 1974, S. 106; Faber, S. 450.

21 BHStA MWi 8246, 26. 9.33; MK 15528, 26.9.33.

22 Vgl. E. Stockhorst, Fünftausend Köpfe. Wer war was im Dritten Reich, 1967, S. 85.

23 Domröse, S. 106. 
rung bildeten. ${ }^{24}$ Auf diese Weise verstärkten die Gelder, die noch von der Republik zur Verfügung gestellt worden waren, den beschäftigungspolitischen Wirkungsgrad des 2. Reichsgesetzes zur Verminderung der Arbeitslosigkeit vom 21. September 1933, nach seinem Urheber auch 2. ReinhardtProgramm genannt. ${ }^{25}$ Der Zweck dieses Gesetzes bestand nach den Worten Hitlers darin, die bisherigen Erfolge der nationalsozialistischen Politik der Arbeitsbeschaffung über den Winter 1933/34 zu bewahren, denn: „Wir gehen einem sehr schweren Winter entgegen. Wir haben von etwas über 6 Millionen Arbeitslosen in acht Monaten über $2 \frac{1}{4}$ Millionen in die Produktion zurückgeführt. Unsere Aufgabe ist, wenn irgend möglich, zu verhindern, daß im Winter ein Absinken eintritt. Im Frühjahr wollen wir dann mit dem neuen Generalangriff gegen die Arbeitslosigkeit beginnen." 26

Andererseits beeilten sich auch einige „Letztempfänger" der Westhilfe, die rechtzeitig den neuen Stil der Wirtschaftspolitik durchschaut hatten, von sich aus eine den nationalsozialistischen Machthabern genehme und für ihre Gewinninteressen vorteilhafte anderweitige Verwendung bereits bewilligter, aber noch nicht ausgegebener Gelder vorzuschlagen. So stand der Handwerkskammer der Pfalz im Juli 1933 noch ein Betrag von 36000 RM aus der RWH 1931 zur Verfügung, der ursprünglich zur Förderung der Bildungseinrichtungen und der Absatzorganisation der Handwerker gedacht war. Nun aber unterbreitete der Verband den Vorschlag, aus diesem Fonds 20000 RM abzuzweigen, um im Zusammenhang mit der Begebung von Ehestandsdarlehen ${ }^{27}$ „Modellschauen“ veranstalten zu können, auf denen ,einem großen Käuferkreis einfache, geschmackvolle und dennoch preiswerte Einrichtungsgegenstände und Hausgeräte" vorgeführt werden könnten. Weitere 10000 RM sollten zur Werbung für ein „Hilfswerk für das deutsche Handwerk“ in Verbindung mit dem Reichsministerium für Volksaufklärung und Propaganda ausgegeben werden. ${ }^{28}$ Welche Bedeutung die nationalsozialistische Regierung der von der Republik erfundenen und aufgebauten Westhilfe beilegte, erhellt aus der im Herbst 1933 vom Reichsinnnenminister und vom Reichsrechnungshof durchgeführten Úberprüfung des Verbleibs aller Gelder, die im Rahmen der Westhilfe zwischen 1927 und 1932 ausbezahlt wor-

${ }^{24}$ Vgl. z. B. GLA 233, Nr. 26042, 5. 12.33; MA 106080, 12.5.34; MA 106079, 28.2., 15.5.33.

25 RGBl. I, 1933, S. $651 \mathrm{f}$.

${ }^{26}$ M. Domarus, Hitler. Reden und Proklamationen 1932-1945, Bd. 1, 1965, S. 322f.; ferner B. Ries, Die Finanzpolitik im Deutschen Reich von 1933-1935, Diss. Freiburg 1964, S. 30.

27 Diese Ehestandsdarlehen - im Durchschnitt 600 RM - vergab der Staat im Rahmen des Gesetzes zur Verminderung der Arbeitslosigkeit vom 1.6.1933 an Arbeiterinnen, die nach der Eheschließung ihren Arbeitsplatz aufgaben. Zum wirtschafts- und bevölkerungspolitischen Hintergrund siehe Mason, S. 51.

28 BHStA MWi 8246, 18.7.33. 
den waren. Die Prüfer fahndeten dabei nicht allein nach gehorteten Beträgen, die man für die nationalsozialistische Politik der Arbeitsbeschaffung hätte nutzbar machen können, sie trugen obendrein einen Überblick über die beschäftigungspolitischen Auswirkungen dieses regionalen Förderungsprogrammes zusammen. ${ }^{29}$

\section{Die Westhilfe als Instrument der Saarpropaganda}

Bereits am 5. April 1933 teilte Hermann Göring, der sich inzwischen des Innenministeriums in Preußen bemächtigt hatte, allen preußischen Ressorts und dem noch amtierenden Reichskommissar v. Papen mit, es sei ,,unbedingt erforderlich“, die Westhilfe auch im Jahre 1933 fortzusetzen, ,und zwar nicht nur wegen der aus der Zerreißung der Grenzen und den Kriegsfolgen sich ergebenden und fortbestehenden Beeinträchtigungen der Lebens- und Wirtschaftsbedingungen dieses Gebietes, sondern insbesondere auch wegen der 1935 bevorstehenden Abstimmung im Saargebiet, die eine Steigerung der besonderen Fürsorge für dieses Gebiet zur politischen Notwendigkeit macht." ${ }^{30}$ Nun hatte sich schon das zweite Kabinett Brünings darum bemüht, im Hinblick auf die Saarabstimmung die Mittel der RWH 1932 so zu verwenden, daß kein allzu großes Gefälle im wirtschaftlichen Wohlstand zwischen dem Saargebiet und den angrenzenden deutschen Landschaften entstehen würde. Offensichtlich türmten sich aber jetzt, nach der nationalsozialistischen Machtergreifung, nicht allein wirtschaftliche Schwierigkeiten an der Saarzollinie auf. In seinem Rundschreiben wies Göring nämlich darauf hin, es scheine ratsam, ,durch eine verstärkte Fortführung der Westhilfe den im Saargebiet sich regenden fremden Einflüssen wirksam entgegenzutreten, welche gegen die Regierung der nationalen Erhebung Stimmung zu machen versuchen." ${ }^{\text {"31 }}$ Mit den fremden Einflüssen meinte Göring wohl kaum die Saarpropaganda der Franzosen. Als wesentlich gefährlichere Gegner des nationalsozialistischen Deutschlands erwiesen sich die deutschen Emigranten, die nach der Machtübernahme ins Saargebiet geflohen waren, wo sie auf ein baldiges Ende des Dritten Reiches hofften. Sie waren Augenzeugen der Vorgänge, welche die "nationale Revolution“ begleitet hatten, viele von ihnen kannten bereits den Alltag nationalsozialistischer Machtausübung, und sie trafen in ihrem Exil auf eine deutsche Bevölkerung, bei der es keine Schwierigkeiten in der Verständigung gab, und die obendrein einen vergleichsweise hohen Anteil der Arbeiterschaft aufwies und überwiegend dem

29 Vgl. ebd., Nachweisliste; GLA 233, Nr. 26042, 22. 5., 5. 10., 5. 12. 33.

30 GStA Rep. 90, Nr. 1128, 5.4.33.

31 Ebd. 
katholischen Bekenntnis angehörte. ${ }^{32}$ Da schon während der „Kampfzeit“ innerhalb des Reiches Wahlgebiete, in denen entweder die Arbeiterbevölkerung vorherrschte oder die katholische Konfession dominierte, am längsten dem Werben der Nationalsozialisten widerstanden hatten, konnte sich Hitlers Regierung nicht mit dem Versuch begnügen, die Bewohner des Saargebietes durch wirtschaftliche Aufbauleistungen im Grenzbezirk zu beeindrukken. Sie mußte sich vielmehr bemühen, den Warnungen der Emigranten vor dem politischen System des Dritten Reiches entgegenzuwirken. Als Instrument der Saarpropaganda blieb das Westprogramm daher nur tauglich, wenn es gelang, die wirtschaftliche Reichswesthilfe in eine politische umzuwandeln.

Allerdings versprach noch im Frühjahr 1933 die rasche Beseitigung der Massenarbeitslosigkeit die beste Werbewirkung für das nationalsozialistische Regime. Die Behebung der Unterbeschäftigung entwickelte sich gerade auch in den Westgebieten zu einer Schicksalsfrage der neuen Regierung. Infolge der politischen Umwälzung und der finanziellen Engpässe verzögerte sich jedoch die Verabschiedung des Reichshaushaltes für das Rechnungsjahr 1933, so daß die Geltung des für 1932 erlassenen Haushaltsgesetzes über den 31. März 1933 hinaus um drei Monate verlängert werden mußte. ${ }^{33}$ Frühestens im Juli konnte also die Reichsregierung einen neuen Abschnitt in der Westhilfe einleiten und verkünden. Da aber andererseits im Saargrenzgürtel ungefähr 15000 Arbeitslose gezählt wurden, die vor dem Eintreten der Wirtschaftskrise im Saargebiet beschäftigt worden waren, ${ }^{34}$ entschloß sich Reichsinnenminister Frick, der Aufforderung Görings zur Fortsetzung des Westprogramms durch eine Sofortmaßnahme zu begegnen. $\mathrm{Zu}$ diesem Zweck benutzte er ein Instrument, das er dem Arbeitsbeschaffungsprogramm v. Papens entlehnt hatte. Um im Tiefpunkt der Weltwirtschaftskrise Hauseigentümer zur Vornahme arbeitsintensiver Reparaturen an ihren Häusern und in ihren Wohnungen anzuregen, hatte der parteilose Arbeitsminister in v. Papens Reichskabinett, Dr. Hugo Schäffer, vorgeschlagen, den „Bauherren“ zinsgünstige Reichsdarlehen zur Verfügung zu stellen, sobald sie bereit wären, eine gewisse finanzielle Eigenleistung zu erbringen. Aus den Darlehensrückflüssen sollten dann wieder neue Kredite vergeben werden. Dieser Gedanke Schäffers war im Papen-Plan vom September 1932 verwirklicht worden. Damals hatte das Reich 50 Millionen als Beihilfe für die In-

32 Vgl. E. Matthias (Hg.), W. Link (Bearb.), Mit dem Gesicht nach Deutschland. Eine Dokumentation über die sozialdemokratische Emigration, 1968, S. 74; G. Lewy, Die katholische Kirche und das Dritte Reich, 1964, S. 203 f.; H.-W. Herrmann, Das Schicksal der Juden im Saarland, in: Dokumentation zur Geschichte der jüdischen Bevölkerung in Rheinland-Pfalz und im Saarland von 1800 bis 1945, Bd. 6, 1974, S. 269 f.

33 BHStA MK 15 528, 30.3.33.

34 BA R 43 II/127 a, Bl. 15. 
standsetzung und den Umbau von Wohnungen zur Verfügung gestellt, wobei jeweils ein Fünftel der Reparaturkosten als Subvention in Darlehensform an die Hauseigentümer fließen sollte. ${ }^{35}$ Die Nationalsozialisten erkannten nicht allein den beschäftigungspolitischen Wert solcher Instandsetzungsarbeiten, sie nahmen auch den finanzpolitischen Vorteil wahr, der in der, induzierten Kapitalbildung" lag, also in der gleichzeitigen Heranziehung fester Quoten privaten Geldkapitals zu den staatlichen Subventionen. ${ }^{36}$ Daher vergrößerten sie bereits im Februar 1933 mittels Notverordnungen den finanziellen Spielraum der Reichsdarlehen zu einem Höchstbetrag von 100 Millionen RM. Gleichzeitig dehnten sie den Geltungsbereich dieser Finanzierung auf landwirtschaftliche Gebäude aus. ${ }^{37}$

Um nun im Saargrenzgürtel einen zusätzlichen Anreiz zu schaffen, solche Reparaturen an Wohnungen und Gebäuden vorzunehmen, stellte Frick eine Million RM als ,,erweiterte Reichswesthilfe 1933“ bereit. Mit dieser Summe sollten in einem Grenzstreifen von $20 \mathrm{~km}$ Tiefe parallel zur Zollgrenze zwei Fünftel - also doppelt so viel wie im übrigen Reichsgebiet - der Instandsetzungskosten abgedeckt werden. Die Landesbehörden wurden angewiesen, diese Mittel unbedingt noch im Rechnungsjahr 1933 auszugeben. Auf diese Weise hoffte der Minister, die zahlreichen Bauhandwerker beschäftigen zu können, die an der Saar ,,abgebaut" worden waren. ${ }^{38}$ Diese Hilfsaktion wurde im Zuge des ersten Reinhardt-Programms ausgebaut, das auf dem Gesetz zur Verminderung der Arbeitslosigkeit vom 1. Juni 1933 beruhte. Nunmehr gewährte das Reichsarbeitsministerium Darlehen in Höhe von einer Million RM zur zusätzlichen Förderung der Instandsetzung von Wohnhäusern und bäuerlichen Wirtschaftsgebäuden im Saargrenzgürtel. Doch handelte es sich bei diesem Betrag nicht um Etatmittel, sondern um $\mathrm{Zu}$ schüsse, die im Wege der Vorfinanzierung auf Wechselbasis in Gestalt der sogenannten „Arbeitsschatzanweisungen“ durch die Deutsche Bau- und Bodenbank aufgebracht worden waren. ${ }^{39}$ Im Rahmen des zweiten ReinhardtProgramms gelang es Reichsarbeitsminister Seldte, zur Bekämpfung der saisonalen Arbeitslosigkeit im Grenzbezirk nochmals 1,9 Millionen RM ,Instandsetzungsmittel" flüssig zu machen. ${ }^{40}$

Schon bei der Durchführung des ersten Reinhardt-Programms zeigte sich

35 Vgl. Marcon, S. 205f.; RGBl. I, 1932, S. 428.

36 K. Schiller, Arbeitsbeschaffung und Finanzordnung in Deutschland, 1936, S. 56.

37 RGBI. I, 1933, S. 79.

38 GStA Rep. 90, Nr. 1128, 22.4.33, 26.3.34.

39 Ebd., 19.10.33; ferner RGBl. I, 1933, S. 323.

40 BHStA MWi 8246, 14.2.34. Von den insgesamt 2,5 Millionen RM ,zusätzlicher Instandsetzungsmittel" entfielen $584000 \mathrm{RM}$,,für einen etwa $25 \mathrm{~km}$ breiten Grenzstreifen der bayerischen Ostmark nördlich der Donau - insbesondere für die Instandsetzung und die Modernisierung von Gasthäusern." 
jedoch, daß viele Gemeinden in der Grenzzone derart verschuldet waren, daß sie selbst die Kredite des Arbeitsbeschaffungsprogramms, die mit günstigen Zins- und Tilgungsbedingungen versehen worden waren, nicht aufnehmen konnten. ${ }^{41}$ Die wirtschaftliche Entwicklung des Grenzgebiets erforderte daher eine weitere Senkung der Kommunallasten. Úberdies wünschte das Innenministerium keine allzu starke Aufsplitterung der Reichsbeihilfen, wie sie bei den Instandsetzungsarbeiten der ,erweiterten $\mathrm{RWH}^{\text {“ notwendiger- }}$ weise in Kauf genommen werden mußten. Im Interesse der Saarpropaganda erstrebte es vielmehr die „Förderung großer Vorhaben“. ${ }^{42}$ Diese Gründe boten dem Reichsinnenminister eine Rechtfertigung, um auch für das Rechnungsjahr 1933 eine ,ordentliche“ Westhilfe auf der Basis verlorener Zuschüsse oder zumindest zinsloser Darlehen zu beantragen. Bei der Aufstellung des neuen Haushaltsplans forderte Frick deshalb für diesen Zweck die Bereitstellung von 8 Millionen RM. Gleichzeitig aber lenkte er die Aufmerksamkeit der Reichsregierung auf die schwierige Lage des Grenzlandes Baden. Insbesondere verlangte er die Ausschüttung von Haushaltsmitteln zur Unterstützung der Stadt Baden-Baden, deren Kurbetrieb immer weiter zurückgehe. Das Gesuch der Stadtverwaltung nach einer Wiedereröffnung ihrer Spielbank habe er nämlich aus ,,ethisch-moralischen Bedenken“" abschlagen müssen. ${ }^{43}$ Reichsfinanzminister Schwerin von Krosigk begegnete diesen finanziellen Anforderungen, indem er auf die Belastungen verwies, die mit den Programmen der Arbeitsbeschaffung auf den Reichshaushalt zukämen. ${ }^{44}$ Reichskanzler Hitler unterband freilich ein langes Feilschen zwischen den beiden Ressorts, indem er eine schnelle Entscheidung herbeiführte. Er setzte sich über die ethisch-moralischen Bedenken seines Innenministers hinweg und erlaubte den bedeutenden und von einem internationalen Publikum besuchten Kur- und Badeorten die Errichtung öffentlicher Spielbanken. ${ }^{45}$ Schwerin von Krosigk hingegen verpflichtete sich, nach Möglichkeit die Aufwendungen für Bahnbauten in der Grenzzone und die immer noch notwendigen Subventionen für den Bleierzbergbau in Mechernich aus dem Westprogramm herauszunehmen und auf das allgemeine Programm der Arbeitsbeschaffung zu übertragen. ${ }^{46}$

Nach dieser Aussonderung von Ansprüchen einigten sich die beiden Minister auf eine RWH 1933 in Höhe von 6 Millionen RM, die Frick bereits Mitte Juli

41 BA R 43 II/127a, Bl. 15.

${ }^{42}$ MK 15 528, 30.3.33.

43 BA R 43 II/127a, Bl. 15. Die öffentlichen Spielbanken waren in Deutschland 1872 geschlossen worden.

44 Ebd.

45 Ebd.; ferner RGBl. I, 1933, S. 480: Gesetz über die Zulassung öffentlicher Spielbanken vom 14.7.1933.

46 Ebd., Bl. 16. 
verteilen konnte. Zunächst sollten 1,5 Millionen für kulturelle Maßnahmen verwendet werden. Nach Abzug einer Kassenreserve von $10 \%$ sollten von den verbleibenden 4,5 Millionen, welche die wirtschaftliche Reichswesthilfe umfaßte, 1,481 Millionen der kommunalen Lastensenkung und 2,569 Millionen der Arbeitsbeschaffung zugeführt werden. Alle Beträge wurden nach dem Schlüssel der Bevölkerungszahl in den Grenzdistrikten auf die Länder verteilt: Bayern erhielt jeweils 54\%, Preußen $37 \%$ und Oldenburg 9\%. ${ }^{47}$ Beim Erlaß der Richtlinien für die Verwendung dieser Mittel stützte sich Frick auf die Vorschriften des bisherigen Westprogramms. Die Vergabe von Einzelsubventionen an Privatfirmen sollte also weiterhin unterbleiben. Den ausführenden Landesbehörden und den „Letztempfängern“ schärfte der Minister ein, die Mittel der Westhilfe dürften künftig nur noch im Saargrenzgürtel, der einen $30 \mathrm{~km}$ breiten, parallel zur Zollgrenze verlaufenden Streifen umschließe, verwendet werden. Dabei solle die Zone I (0-20 km) vergleichsweise stärker berücksichtigt werden als die Zone II $(20-30 \mathrm{~km})$. Wenn es allerdings um die Fertigstellung von Wasserleitungen und Straßenbauten, um die Durchführung von Meliorationen, um die Anlage von Obstkulturen und um die Einrichtung von Absatzorganisationen gehe, könnten auch die benachbarten luxemburgisch-belgischen und die elsässischen Grenzgebiete bis $\mathrm{zu}$ einer Tiefe von $20 \mathrm{~km}$ in die Reichswesthilfe einbezogen werden. ${ }^{48}$ Vergleicht man die Struktur der Verwendung der Mittel bei der RWH 1933 mit derjenigen ihrer „republikanischen“ Vorgänger, so fällt sofort der relativ hohe Anteil auf, der im Etatjahr auf kulturelle Zwecke entfiel. Allerdings verstand das Dritte Reich unter „kultureller Fürsorge“ nicht dasselbe wie die Republik von Weimar. Zu seinen kulturellen Maßnahmen zählte z.B. der Ausbau des Radiosenders Kaiserslautern ,im Hinblick auf die Saarabstimmung““ ${ }^{49}$ Goebbels, seit dem 31. März 1933 Reichsminister für Propaganda und Volksaufklärung, erkannte klar die Bedeutung des Rundfunks für die Beeinflussung der Stimmberechtigten an der Saar. ${ }^{50}$ Überdies wußte er, daß einerseits die Franzosen einen leistungsfähigen Sender in Straßburg für die Saarpropaganda einsetzen konnten, daß aber andererseits die Westgebiete des Reiches vom bestehenden deutschen Rundfunknetz infolge der geringen Reichweite der Sender nicht richtig erfaßt wurden. ${ }^{51}$ Auch im Bereich der

47 BHStA MA 105977, 13.7.33.

48 BHStA MA 105981, 21.8.33; MWi 8246, 16.10.33; ferner MWi 7143, 18.12.33 (Ablehnung weiterer Zuschüsse an die Bürstenindustrie in Ramberg/Pfalz, da diese nicht zum ,Saargrenzgürtel“" gehöre).

49 Ebd. MWi 8246, 14.12.33.

50 K. Bartz, Weltgeschichte an der Saar, 1935, S. 57.

51 Ebd., S. 63; H. Pohle, Der Rundfunk als Instrument der Politik. Zur Geschichte des deutschen Rundfunks von 1923/38, 1955, S. 245. 
Kultur wurde deshalb die Bewilligung von Zuschüssen dem Ziel der Saarpropaganda untergeordnet. So wurde das Hilfegesuch des Pfalzorchesters, das die Republik aus Mitteln der kulturellen Westhilfe unterstützt hatte, im Reichsinnenministerium mit der unverbindlichen Erklärung abgewiesen, der Ausbau des Radiosenders Kaiserslautern werde ,,voraussichtlich“ auch dem Pfalzorchester neue Möglichkeiten der Beschäftigung bieten. ${ }^{52}$

Der Vorrang der Saarpropaganda prägte auch die Gestalt der RWH 1934. Für dieses Etatjahr stockte der Finanzminister den Westfonds auf 9 Millionen RM auf. Davon sollte die eine Hälfte dem Saargebiet, die andere dem Saargrenzgürtel zufließen. Von den 4,5 Millionen, die auf die Saar entfielen, kassierte das Reichspropagandaministerium vorab 1,9 Millionen. ${ }^{53} \mathrm{Da}$ die Quote, die der Grenzzone zustand, vorwiegend der kommunalen Lastensenkung diente, ${ }^{54}$ kamen der politischen Führung bald Bedenken, ob bei der Aufteilung des Westfonds die Arbeitsbeschaffung gegenüber der Propaganda nicht eindeutig zu kurz gekommen sei. Eine Vernachlässigung der Beschäftigungspolitik könnte indessen die Folge zeitigen, daß die Wirkungen der Rundfunkpropaganda durch das Stagnieren der Wirtschaft in der von den Saarländern häufig besuchten Grenzzone wieder ausgewischt werden würden. ${ }^{55}$ Anfang September 1934 - also kurz vor dem Eintreten des saisonbedingten Rückganges der Beschäftigung - bemühte sich daher das Reichsinnenministerium um eine beschleunigte Durchführung von ,,arbeitsschaffenden“ Projekten im Grenzgürtel. Mit dem Vermerk „Eilt sehr!“ belehrte Frick die Landesbehörden: „Die Rücksichtnahme auf die Saarabstimmung am 13. Januar 1935 erfordert aber, daß die Mittel der Reichswesthilfe 1934 so eingesetzt werden, daß in den kommenden Monaten bis zum Frühjahr 1935 unter allen Umständen ein Ansteigen der Arbeitslosenziffer im Saargrenzgürtel vermieden wird." ${ }^{\text {56 }}$ Es dürfte außer Zweifel stehen, daß die geschickte Propagandatätigkeit der Nationalsozialisten und die gezielte wirtschaftliche Förderung der dem Saargebiet benachbarten deutschen Bezirke mit dazu beitrug, daß die Saarabstimmung derart eindeutig zugunsten des Dritten Reiches ausfiel: Am 13. Januar 1935 sprachen sich 80,43\% der insgesamt Stimmberechtigten mit $90,76 \%$ der abgegebenen gültigen Stimmen für eine Rückgliederung des Saargebietes an Deutschland aus. ${ }^{57}$ Dieses Er-

52 BHStA MK 15 528, 14.12.33.

53 Ebd. MWi 8247, 18. 5.34; ferner MWi 8246, 27.4.34; M. Zenner, Parteien und Politik im Saargebiet unter dem Völkerbundsregime 1920-1935, 1966, S. $258 \mathrm{f}$.

54 Ebd., 19. 5., 29.8.34.

55 Siehe hierzu Bartz, S. $57 \mathrm{f}$.

56 BHStA MWi 8247, 29.9.34.

57 Siehe hierzu Jacoby, S. 11 f.; Zenner, S. 316 f.; Faber, S. 448. Die Nationalsozialisten selbst schätzten die Wirksamkeit der wirtschaftlichen Werbung im Saargrenzgürtel im Hinblick auf das Ergebnis der Abstimmung hoch ein. Vgl. Bartz, S. 58. 
gebnis beeindruckte die Weltöffentlichkeit, so daß der Rat des Völkerbundes bereits in seiner Sitzung am 17. Januar als Datum der Rückgabe des Saargebietes an das Deutsche Reich den 1. März 1935 bestätigte.

\section{Die Westhilfe als Mittel der Arbeitsbeschaffung in der entmilitarisierten Zone}

Nach dem Ende der politischen und wirtschaftlichen Saarpropaganda offenbarten sich erneut die Schwächen in der Wirtschaftsstruktur der Grenzgebiete im Westen, die aber jetzt durch die Politik der nationalsozialistischen Regierung noch erheblich verschärft wurden. Bereits Ende 1934 benachrichtigte das Reichsinnenministerium die Landesbehörden unter dem Siegel strenger Geheimhaltung davon, daß ,,besondere Notwendigkeiten in letzter Zeit“ dazu geführt hätten, ,,einzelne Industrien aus den Grenzgebieten in das Binnenland zu verlegen und bestimmte Aufträge nur noch an binnenländische Industrien zu vergeben." 58 Hinter dieser geheimnisvollen Andeutung verbarg sich die der Öffentlichkeit noch weitgehend verschlossene Aufrüstung des Dritten Reiches. Der Aufbau und der Betrieb einer Rüstungsindustrie, die nach dem Friedensvertrag untersagt war, vollzog sich natürlich im Landesinnern wesentlich sicherer und geschützter als in den Grenzgebieten, wo Rüstungsbetriebe nicht allein durch verstärkte Spionage- und Sabotagetätigkeit gefährdet wurden, sondern obendrein dem Zugriff rascher militärischer Aktionen der Polen, Tschechen, Franzosen und Belgier ausgesetzt waren. ${ }^{59}$ Der Westen schien als Standort für eine geheime Aufrüstung besonders gefährdet, galt er doch nach internationalem Recht nach wie vor als „Entmilitarisierte Zone“. Diese Zone umfaßte das gesamte linksrheinische Gebiet sowie einen etwa $50 \mathrm{~km}$ breiten Streifen ostwärts des Stromes. Die Bestimmungen des Friedensvertrages von Versailles untersagten Deutschland in diesem Landesteil die Unterhaltung von Streitkräften, die Durchführung militärischer Übungen und die Anlage von Befestigungen. Über rein militärische Belange ausgreifend trafen sie aber auch die Privatwirtschaft empfindlich, z.B. durch ihre restriktive Reglementierung der Sprengstoffund Sprengkörperindustrie, deren Standort in Köln bis 1914 ,,einer der führenden in Europa, ja in der Weltwirtschaft gewesen ist."60

Die Abneigung der Industrie, im ungeschützten Grenzgebiet größere Investi-

58 GStA Rep. 90, Nr. 1128, 9. 12.34. Gleichzeitig wurden in diesem Rundschreiben die Landesbehörden angewiesen, ,aus staats- und grenzpolitischen Gründen“ die Westgebiete durch verstärkte Vergabe von Aufträgen für diese Ausfälle zu entschädigen.

$59 \mathrm{Vgl}$. G. Thomas, Geschichte der deutschen Wehr- und Rüstungswirtschaft, 1966, S. 97.

60 Kuske, S. 125. 
tionen zu tätigen, entsprang schließlich auch den noch nicht lange zurückliegenden Erfahrungen mit den Verkehrsbehinderungen in den Jahren des Ruhrkampfes. ${ }^{61}$ Ähnlich vorsichtig verhielt sich der Staat als Auftraggeber. Im Frühjahr 1935, als im gesamten Reichsgebiet die Baustellen der Reichsautobahn bereits zahlreiche Arbeitskräfte an sich zogen, lehnte das Reichswehrministerium die Inangriffnahme der Teilstrecke zwischen Köln und Aachen ,,aus grundsätzlichen Erwägungen“ „,vorläufig“ ab. ${ }^{62}$ Dabei hatte die Rheinische Provinzialverwaltung bereits 1925 den Bau einer "Autostraße“ von Aachen nach Köln beantragt, und zwar nicht allein aus verkehrspolitischen Erwägungen, sondern gerade auch in der erklärten Absicht, der hohen Arbeitslosigkeit im Raum Aachen und Düren entgegenzusteuern. ${ }^{63}$ Die Besorgnis der militärischen Stäbe, diese Autobahn könne eine feindliche Invasion in das Reichsinnere begünstigen, vermochte jedoch offensichtlich die Aufgabe der Arbeitsbeschaffung in den Hintergrund zu drängen. Schließlich bedurfte es einer Intervention Hitlers, um den Beginn der Bauarbeiten an dieser Strecke der Reichsautobahn im April 1936 sicherzustellen. ${ }^{64}$ Die Erwartungen, welche die Industrie im westlichen Grenzgebiet - Baden eingeschlossen - auf einen entsprechenden Ausgleich durch die Vergabe ,neutraler Aufträge" seitens der Reichspost und der Reichsbahn gesetzt hatte, wurden ebensowenig erfüllt. ${ }^{65}$

Ferner hemmte die nationalsozialistische Außenwirtschaftspolitik die industrielle Entfaltung in der entmilitarisierten Zone, weil sie der dort vorherrschenden Konsumgüterindustrie gleichzeitig die Einfuhr ihrer Rohstoffe und die Ausfuhr ihrer Erzeugnisse durch administrative Maßnahmen verteuerte und erschwerte. Im Gegensatz zur Schwer- und Grundstoffindustrie verfügten die meisten der im Westen ansässigen Industriefirmen nicht über eine so große wirtschaftliche Macht, daß sie sich entweder am Binnenmarkt durch die Forderung höherer Preise für entgangene Exportgewinne hätten schadlos halten können oder daß sie sich im Rahmen einer internationalen Kartellabsprache einen ausländischen Marktanteil hätten sichern lassen können. ${ }^{66}$ Als besonders benachteiligt erwies sich der Wirtschaftsraum Aachen, der durch eine Ballung von Textilunternehmungen und von unrentablen, im Vergleich

61 Mason, S. $73 \mathrm{f}$.

62 B. Vollmer, Volksopposition im Polizeistaat. Gestapo- und Regierungsberichte 1934-1936, 1957, S. 191.

63 K. Kaftan, Der Kampf um die Autobahnen, 1955, S. 65.

64 Vollmer, S. 270f., 373. Freilich hatte inzwischen, nach dem Einmarsch deutscher Truppen ins Rheinland Anfang März 1936, die Remilitarisierung dieser Zone begonnen.

65 Vollmer, S. 191; Verfolgung und Widerstand unter dem Nationalsozialismus in Baden. Die Lageberichte der Gestapo und des Generalstaatsanwalts Karlsruhe 1933-1940, 1976, S. 182.

66 Vgl. D. Doering, Deutsche Außenwirtschaftspolitik 1933-35, 1969, S. 205f.; Verfolgung und Widerstand, S. 186. 
zum Ruhrbergbau wenig produktiven Zechen gekennzeichnet war. $\mathrm{Zu}$ den wirtschaftlichen Strukturschwächen dieser Region gesellten sich in zunehmendem Maße politische Behinderungen der wirtschaftlichen Entwicklung. Vor allem in der Stadt Aachen klagten die Geschäftsleute über den Boykott, den viele ihrer einstmals guten Kunden aus den benachbarten belgischen und niederländischen Grenzgebieten seit der nationalsozialistischen Machtergreifung über sie verhängt hatten. ${ }^{67}$

Im Bereich der Landwirtschaft lösten die Diskriminierung der Juden und deren allmähliche Verdrängung aus dem Wirtschaftsleben Absatzstockungen aus, weil im Westen bis 1933 jüdische Unternehmer im Getreide-, Weinund Viehhandel, in der Rheinpfalz auch im Lederhandel, auf Grund ihrer Fähigkeiten und ihrer Sachkenntnis beträchtliche Marktanteile behauptet hatten. ${ }^{68}$ Insbesondere in Rheinhessen hatten sich jüdische Weinhändler als ein unentbehrliches Bindeglied zwischen Erzeuger und Verbraucher erwiesen, das nun allmählich der Judenverfolgung zum Opfer fiel..$^{69}$ Bereits im Juni 1933 mußte der badische Reichskommissar Wagner auf einer Verbandstagung der landwirtschaftlichen Genossenschaften in Darmstadt - unter unfreiwilliger Würdigung des kaufmännischen Talents der Ausgesperrten und Verfolgten - eingestehen, er habe bei seiner Reise durch die rheinhessischen Weinbaugebiete selbst festgestellt, daß ,wegen der gemeinen Zurückhaltung der jüdischen Händler" der Absatz der Qualitätsweine erhebliche Schwierigkeiten bereite. ${ }^{70}$

Aus allen diesen Gründen wirkte sich die Staatskonjunktur, die nach und nach die deutsche Volkswirtschaft erfaßte ${ }^{71}$, in den Westgebieten nur stark abgeschwächt aus. Im Winter 1935/36 kam es im Rheinland sogar zu einer Wirtschaftskrise, welche die rheinischen Industrie- und Handelskammern, die Banken und die Zivilbehörden einhellig dem entmilitarisierten Status ihrer Region zuschrieben. Die ortsansässige Industrie erhalte nämlich kaum Aufträge der Wehrmacht, die leerstehenden Garnisonen ließen in vielen Städten den Einzelhandel nicht aufkommen, während ein Neubau oder eine

67 Vollmer, S. 46.

68 J. Simmert (Bearb.), Die nationalsozialistische Judenverfolgung in Rheinland-Pfalz 1933 bis 1945, in: Dokumentation, S. 99. Auch in anderen Teilen des Reiches besaßen jüdische Kaufleute im Getreide- und Viehhandel eine solche Schlüsselstellung, die durch die „Arisierung“ zum Schaden der Erzeuger und der Verbraucher erschüttert wurde. Vgl. z. B. R. Thévoz u. a., Pommern 1934/35 im Spiegel von Gestapo-Lageberichten und Sachakten, Bd. 1, 1974, S. $183 \mathrm{f}$.

69 Simmert, S. 115.

70 K. Düwell, Die Rheingebiete in der Judenpolitik des Nationalsozialismus vor 1942. Beitrag zu einer vergleichenden zeitgeschichtlichen Landeskunde, 1968, S. $95 \mathrm{f}$.

71 Siehe hierzu K. Borchardt, Wachstum und Wechsellagen 1914-1970, in: Hb. der Deutschen Wirtschafts- und Sozialgeschichte, Bd. 2, 1976, S. $712 \mathrm{f}$. 
Instandsetzung von Kasernen und anderen militärischen Anlagen das Baugewerbe und das Handwerk beleben könnte. ${ }^{72}$

Die Unzufriedenheit im Westen beschränkte sich indessen nicht auf die Unternehmer, die Landwirte und die Winzer. Obwohl das Lohnniveau der entmilitarisierten Zone deutlich hinter demjenigen vergleichbarer anderer Wirtschaftsregionen des Reiches zurückblieb, ergoß sich der allgemeine Preisauftrieb ungehemmt auch über den Westen. ${ }^{73}$ Die Teuerung erfaßte vor allem die Lebensmittel und rief Schwierigkeiten in der Versorgung der Bevölkerung hervor, die schließlich in der sogenannten ,Brotkrise“ im Winter 1935/ 36 gipfelten. ${ }^{74}$ Daher erstaunt es nicht, wenn sich bereits Ende 1934 die Unternehmer der Eisen- und Metallverarbeitung in der entmilitarisierten Zone, insbesondere im Rheinland, darüber beschwerten, daß ihnen immer mehr junge und tüchtige Facharbeiter von ihren Konkurrenten im übrigen Reichsgebiet durch das Versprechen höherer Löhne abgeworben würden. Um diese Abwanderung zu bremsen, ordnete die Reichsanstalt für Arbeitsvermittlung und Arbeitslosenversicherung an, daß Facharbeiter, die am 15. Januar 1935 ihren Wohnsitz in der entmilitarisierten Zone hatten, nur mit Zustimmung des für diesen Wohnsitz zuständigen Arbeitsamtes ihren Arbeitsplatz wechseln durften. ${ }^{75}$ Solche Auflagen nützten jedoch allenfalls den Unternehmern. Die Lebenshaltung der Arbeiter vermochten sie indessen nicht zu verbessern, wie die folgende geheimgehaltene Meldung der Gestapo aus dem Raum Aachen vom August 1935 bezeugt: ,Wenn berichtet werden muß, daß vielfach die Kumpels bei ihrer schweren Arbeit Brote mit in die Grube nehmen, die nur mit Kraut und Pellkartoffeln belegt sind, und sich kaum noch die billigste Margarinesorte erlauben können, so erscheinen diese Besorgnisse doppelt begründet, zumal diese Leute, die manchmal schon einen unterernährten Eindruck machen, sehen und hören, daß auf der anderen Seite gewisse Kategorien von Staatsbeamten oder Parteifunktionäre sich in ihrer Lebenshaltung nicht die allergeringste Beschränkung aufzuerlegen brauchen." 76

Sozialer Zündstoff ballte sich ferner im Saargrenzgürtel zusammen, wo die Zahl der Arbeitslosen wieder zu wachsen drohte. Die im Zuge der Saarpropaganda angelaufenen Vorhaben der Arbeitsbeschaffung liefen allmählich aus. Zwar wurden im Saargebiet selbst gerade „Arbeitsbeschaffungsmaßnah-

${ }^{72}$ H. Lademacher, Die nördlichen Rheinlande von der Rheinprovinz bis zur Bildung des Landschaftsverbandes Rheinland (1815-1953), in: Petri, Bd. 2, S. 756, 764.

73 Vollmer, S. 255; Verfolgung und Widerstand, S. 186.

74 G. Meinck, Hitler und die deutsche Aufrüstung 1933-1937, 1959, S. 161.

75 F. Syrup, Arbeitseinsatz der Metallarbeiter, in: Reichsarbeitsblatt II, 1937, S. 98f.; ferner R. Hanf, Möglichkeiten und Grenzen betrieblicher Lohn- und Gehaltspolitik 1933-1939, 1975, S. 53 f.

76 Vollmer, S. 256. 
men großen Stils" durchgeführt, doch verhielten sich dort die neuen Machthaber als Arbeitgeber nicht anders als früher die Saarkommission unter dem Völkerbundsmandat: Auch sie beseitigten die Arbeitslosigkeit an der Saar auf Kosten der Quote der Beschäftigten im angrenzenden deutschen Gebiet. ${ }^{77}$ Außerdem machten sie die Zuwanderung auswärtiger Arbeitskräfte für das Saarland genehmigungspflichtig, eine Maßnahme, die sie, hauptsächlich um der Landflucht vorzubeugen, sonst nur für Berlin, Hamburg und Bremen verhängt hatten. ${ }^{78}$ Das Einkommensgefälle zwischen dem Saargebiet und dem Saargrenzgürtel nahm schließlich noch zu, als Gauleiter Bürckel sich mit Erfolg dafür einsetzte, daß das vergleichsweise hohe Lohnniveau der Firma Röchling auf alle saarländischen Industriebetriebe ausgedehnt wurde. ${ }^{79}$ Noch am 12. Oktober 1935 beklagte sich der Kreisleiter der NSDAP in Kusel/Westpfalz beim Reichsinnenminister über die weiterhin anhaltende Erwerbslosigkeit unter den früheren Saargängern, ,,da aus politischen Gründen die erwerbslosen Volksgenossen des Saargebiets zuerst in Arbeit gebracht werden müssen." 80

Um einen jähen Abbruch der bisherigen Beschäftigungspolitik an der Westgrenze zu vermeiden und die Fortführung der arbeitsintensiven Bauvorhaben zur Verbesserung der Infrastruktur zu gewährleisten, ${ }^{81}$ bemühte sich Frick, auch für das Etatjahr 1935 einen Westfonds von 4,5 Millionen RM durchzusetzen. Finanzminister Schwerin von Krosigk erklärte sich schließlich mit einer RWH 1935 in Höhe von 3 Millionen einverstanden, die zu etwa einem Drittel zur kommunalen Lastensenkung und zu zwei Dritteln zur Förderung der Wirtschaft eingesetzt wurden. ${ }^{82}$ Da auch im Verlauf des Jahres 1935 die wirtschaftliche Entwicklung in vielen Gebieten der entmilitarisierten Zone hinter dem durchschnittlichen Wachstum der deutschen Volkswirtschaft zurückblieb, ${ }^{83}$ trat Frick, der inzwischen auch das Innenministerium Preußens verwaltete, in erneute Verhandlungen mit Schwerin von Krosigk über die weitere Gestaltung des Westprogramms ein. ${ }^{84}$ Nachdrücklich bemühte sich vor allem der Reichsstatthalter in Bayern, von Epp, um die Schaffung einer

77 BHStA MWi 8248, 13.5.35.

78 Mason, S. 54. Diese Zuzugssperre vermochte die Landflucht jedoch nicht zu drosseln. $\mathrm{Zu}$ den hohen Wanderungsverlusten der ländlichen Bevölkerung im Südwesten Deutschlands siehe D. Schoenbaum, Die braune Revolution. Eine Sozialgeschichte des Dritten Reiches, 1968, S. 221.

79 Hüttenberger, S. 140.

80 BHStA MWi 8247, 12.10.35.

81 Am 5. 3. 1934 hatte der Präsident des Reg.-Bez. Aachen der Gestapo in Berlin berichtet, es lägen eine Reihe von „,baureifen“ Anträgen zur Errichtung von Wasserleitungen und zum Straßenbau vor, für welche Darlehensmittel fehlten. Vollmer, S. 62.

82 BHStA MWi 8247, 20. 2.35; MWi 8248, 27.4.35.

83 Vgl. Braun, S. 84.

84 BHStA MK 15529, 21.1.36. 
RWH 1936, ,... da ein plötzliches Abreißen der Arbeitsbeschaffungsmaßnahmen durch die öffentliche Hand zu schweren Belastungen der Arbeitsmarktlage in der Pfalz führen müßte, die ohnedies gegenüber der allgemeinen Lage im übrigen Deutschen Reich wenig günstig erscheint. “85 Diese auffallende Aktivität Epps hatte wohl das Verhalten des Gauleiters Bürckel ausgelöst, der, „ohnehin schon oppositionell gegenüber München“, inzwischen in der Pfalz eine eigenwillige Politik verfolgte, ,,die erheblich mehr Affinität zu sozialistischen Tendenzen hatte, als man in München gutheißen wollte." ${ }^{\circ 86}$

Da Schwerin von Krosigk aus fiskalischen Gründen der Fortführung der Westhilfe ablehnend gegenüberstand, versuchte Frick, wenigstens die Überschüsse der Reichsanstalt für Arbeitsvermittlung und Arbeitslosenversicherung als Darlehen zur Schaffung von Arbeitsplätzen im Westen zu nutzen, obwohl er sich der im Vergleich zu den verlorenen Zuschüssen aus der Westhilfe erheblich geringeren beschäftigungspolitischen Wirksamkeit dieser Kredite durchaus bewußt war. ${ }^{87}$ Vorsichtshalber forderte das Reichsinnenministerium die Landesbehörden auf, alle noch vorhandenen Mittel aus dem Westfonds auszugeben, da die Übertragbarkeit dieser Gelder auf eine künftige RWH 1936 nicht gesichert sei. ${ }^{88}$ Dabei zeigte sich, daß selbst aus der RWH 1930 noch Gelder zur Verfügung standen, die bisher nicht in voller Höhe in Anspruch genommen worden waren, oder die nun durch allmähliche Tilgung ,zurückflossen“, weil sie seinerzeit als zinslose Darlehen vergeben worden waren. So konnten im Regierungsbezirk Aachen 15000 RM, die ursprünglich für die Eröffnung einer Beratungsstelle des Deutschen Handwerksinstituts bewilligt worden waren, ,endgültig“ einer Handwerksgenossenschaft zur Anschaffung von Maschinen und zur Ausbildung von Lehrschweißern zugeteilt werden. ${ }^{89}$ In Rheinhessen konnten $80000 \mathrm{RM}$, die aus „Darlehensrückflüssen“ der RWH 1930 stammten, in Zuschüsse zum Umbau von Wohnungen umgewandelt werden, die $20 \mathrm{v}$. H. der gesamten Baukosten decken sollten. Dank dieser Finanzierungsquelle konnten im Winter 1936/37 zum Nutzen des lokalen Handwerks, das über Auftragsmangel klagte, Bauvorhaben begonnen werden, deren Gesamtkosten fast 400000 RM erreichten..$^{90}$

Ein solches Ergebnis war der politischen Führung auch noch im Jahre 1937 willkommen, weil die Rüstungsausgaben und die Politik des Vierjahresplans hauptsächlich bei der mittleren und großen Industrie zu Buche schlugen,

85 Ebd., 11. 1.36.

86 Domröse, S. 308.

87 BHStA MK 15529, 21.1., 14.4.36.

88 BA R 2/11 847, 26.3., 4. 4.36; BHStA MWi 8247, 14.2.36, MA 105981, 6. 2. 36.

89 BA R 2/11 847, 21.4.36.

90 Ebd., 7. 9., 8. 10., 24. 10.36. 
nicht aber bei den Handwerkern, in deren Reihen die NSDAP schon vor der Machtübernahme zahlreiche und treue Anhänger gefunden hatte. ${ }^{91}$ Festzuhalten bleibt indessen, daß die Erfolge der Reichswesthilfe, mit denen sich die Nationalsozialisten in der Öffentlichkeit brüsteten, ${ }^{92}$ unmittelbar auf ein regionales Förderungsprogramm zurückgingen, das von den Politikern der den neuen Machthabern so verhaßten „Systemzeit" erfunden und in Gang gesetzt worden war.

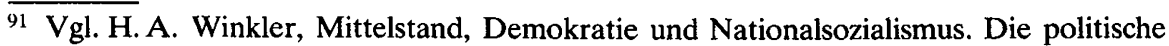
Entwicklung von Handwerk und Kleinhandel in der Weimarer Republik, 1972, S. $183 \mathrm{f}$.

$92 \mathrm{Vgl}$. Bayern im ersten Vierjahresplan. Denkschrift der Bayerischen Landesregierung zum 9. März 1937, 1937, S. 395. 